\title{
Image Segmentation and Analysis via Multiscale Gradient Watershed Hierarchies
}

\author{
John M. Gauch, Member, IEEE
}

\begin{abstract}
Multiscale image analysis has been used successfully in a number of applications to classify image features according to their relative scales. As a consequence, much has been learned about the scale-space behavior of intensity extrema, edges, intensity ridges, and grey-level blobs. In this paper, we investigate the multiscale behavior of gradient watershed regions. These regions are defined in terms of the gradient properties of the gradient magnitude of the original image. Boundaries of gradient watershed regions correspond to the edges of objects in an image. Multiscale analysis of intensity minima in the gradient magnitude image provides a mechanism for imposing a scale-based hierarchy on the watersheds associated with these minima. This hierarchy can be used to label watershed boundaries according to their scale. This provides valuable insight into the multiscale properties of edges in an image without following these curves through scale-space. In addition, the gradient watershed region hierarchy can be used for automatic or interactive image segmentation. By selecting subtrees of the region hierarchy, visually sensible objects in an image can be easily constructed.
\end{abstract}

Index Terms - Image segmentation, multiscale image analysis, watershed regions.

\section{INTRODUCTION}

$\mathbf{M}$ ULTISCALE image analysis and image segmentation play an important role in many computer vision applications. Together, they provide an indication of where visually sensible objects in an image are located and also information about their relative size or importance. With this information, it is possible to perform quantitative measurements of object properties such as size, shape, position, and orientation, and to accomplish higher level vision tasks such as object recognition. Early multiresolution methods utilized somewhat ad hoc resolution reduction schemes, but they produced compact image descriptions which were useful for a number of computer vision tasks [7], [38]. Gaussian blurring was later introduced to study the scale-space behavior of intensity extrema in signals and images [26], [44]. One of the attractive properties of this technique is that images simplify in a well behaved manner. For example, Gaussian blurring does not create any zero crossings as resolution is reduced [1], [45].

The multiscale behavior of a number of image features have been examined. Paths traced by intensity extrema through

Manuscript received March 31, 1995; revised November 1, 1996. This work was supported in part by a grant from the Whitaker Foundation, the National Science Foundation (CDA-9 401 021), and the National Cancer Institute (CA$42165)$.The associate editor coordinating the review of this manuscript and approving it for publication was Prof. William E. Higgins.

The author is with the Department of Electrical Engineering and Computer Science, University of Kansas, Lawrence, KS 66045 USA (e-mail: jgauch@eecs.ukans.edu).

Publisher Item Identifier S 1057-7149(99)00220-1. scale-space have been used to study one-dimensional (1-D) signals [11], [44]. The image stack is the result of similar analysis of critical points in two-dimensional (2-D) images [26]. This multiscale image representation has also been used for image segmentation [27]. The multiscale behavior of greylevel blobs (defined relative to intensity extrema) has been used to develop a scale-space primal sketch and used for image segmentation and analysis [28]. The performance of both of these segmentation techniques suffered somewhat because edge information was not explicitly included. Edges defined by Laplacian of Gaussian zero crossings [30] and zeros of directional derivatives [8], [20] have been traced through multiple scales in an attempt to identify significant object boundaries and deal with image noise [2], [22]. One problem with these methods is the difficulty of retaining connected edge segments through scale-space. A second difficulty is constructing object regions from these boundaries.

The multiscale behavior of intensity ridges and valleys in an image have also been studied [15]. Ridge tops and valley bottoms were defined in terms of the local differential geometry of the image (extrema of level curve curvature) and followed through scale-space. This process results in well localized ridges and valleys but involves costly multiscale curve following. To simplify this analysis, a representation for ridge tops and valley bottoms based on watershed boundaries was utilized. The drainage patterns of simulated rainfall on an image can be used to partition an image into watershed regions called hills and dales [9], [31]. The boundaries of hills correspond to ridge tops and the boundaries of dales correspond to valley bottoms, so multiscale watershed analysis provides an alternative method to study the scale-space behavior of ridges and valleys in an image [16].

Mathematical morphology provides a powerful set of nonlinear image analysis tools which can be applied in a wide variety of situations [13], [21], [41]. For example, images can be segmented into visually sensible regions by finding the watershed regions in a gradient magnitude image [32], [42]. Oversegmentation is a well-known difficulty with this approach, which has led to a number of approaches for merging watershed regions to obtain larger regions corresponding to objects of interest [17], [19], [34], [39], [40]. The development of morphological scale-space operations [10], [23] has also made it possible to study the multiscale behavior of watershed regions [24], [25]. One advantage of this approach is that no new intensity extrema (or corresponding watershed regions) are created as scale is increased. In spite of recent speed improvements [5], [14] the mathematical morphology scalespace approach remains computationally demanding. 
The focus of this paper is on the multiscale analysis of watershed regions using a Gaussian filtering based scale-space. In Section II, we review methods for computing watershed regions and describe how multiscale analysis of the intensity extrema in an image can be used to define a scale-based hierarchy on watershed regions. We then show how the closed boundaries of these watershed regions can then be labeled with a visually meaningful measure of their importance. In Section III, we show how multiscale analysis of intensity extrema in the gradient magnitude image can be used to define a hierarchy on the corresponding watershed regions. We then show how this region hierarchy can be used to label gradient watershed boundaries according to their scale, thereby providing insight to the relative importance of edges in an image without actually following these curves through scalespace. Finally, we describe how the gradient watershed region hierarchy can be used for automatic and interactive image segmentation. By selecting subtrees of the region hierarchy, visually sensible objects in an image can be easily constructed.

\section{INTENSITY WATERSHED HIERARCHIES}

Work on watersheds began over a hundred years ago when Cayley and Maxwell [9], [31], described how smooth surfaces could be decomposed into hills and dales by studying the critical points and slope lines of a surface. By viewing intensity in an image as elevation and simulating rainfall, it is possible to decompose an image into watershed regions. Since this image decomposition is useful for a number of purposes, a number of methods to find watershed regions and their boundaries have been devised.

Early watershed algorithms were developed to process digital elevation models and were based on local neighborhood operations on square grids [12], [29], [37]. Improved gradient following methods were subsequently devised to overcome problems with intensity plateaus and square pixel grids [6], [18], [35]. Other approaches use "immersion simulations" to identify watershed regions by flooding the image with water starting at intensity minima [3], [4], [42]. Here, a variety of data structures including priority ordered queues and hierarchical queues are used to efficiently select pixels to add to watershed regions.

The level of activity in the area of watershed identification reflects to some degree the difficulty of this task. Much of the complexity of current techniques is indirectly due to pixel quantization. For example, if a $5 \times 5$ region of uniform intensity appears in the image, central pixels will have a gradient of zero. In order to determine if this region corresponds to a local maxima or minima or is part of a hillside in the image, all of the neighbors of the flat region must be examined. We avoid this complexity by working with Gaussian smoothed floating point images. This removes all regions with uniform intensity (except in the case of an input image with only one intensity value). We can then use fast and simple gradient following algorithms based on local pixel properties to identify watershed regions.

Our investigation of watersheds has three phases. First, we describe how watersheds and their boundaries can be

\begin{tabular}{|l|l|l|l|l|l|}
\hline 80 & 73 & 71 & 79 & 82 & 80 \\
\hline 74 & 69 & 66 & 68 & 71 & 73 \\
\hline 68 & 67 & 65 & 67 & 69 & 71 \\
\hline 74 & 70 & 66 & 68 & 62 & 67 \\
\hline 79 & 71 & 68 & 63 & 61 & 66 \\
\hline 82 & 80 & 70 & 66 & 64 & 68 \\
\hline
\end{tabular}

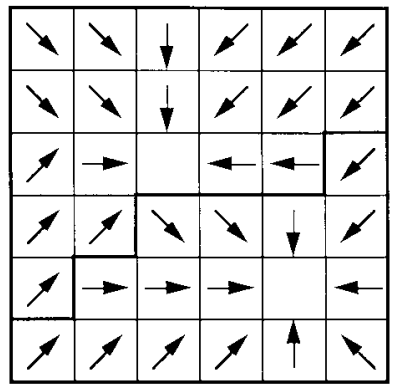

Fig. 1. Simple example of watershed regions and their boundaries. Gradient vectors indicate the direction toward the lowest value 8-neighbor at each point. All points drain to intensity minima 61 or 65 , defining two watershed regions. The watershed boundary is shown in bold.

computed for 2-D images. Then, we impose a scale-based hierarchy on watershed regions based on the behavior of critical points in the image under Gaussian blurring. Finally, we use this watershed hierarchy to assign importance related scales to watershed boundary segments. These techniques extend naturally to three dimensions, but this is not described in this paper.

\section{A. Watersheds and Their Boundaries}

Watersheds are traditionally defined in terms of the drainage patterns of rainfall. Regions of terrain that drain to the same point are defined to be part of the same watershed. The same analysis can be applied to images by viewing intensity as height. In this case, the image gradient is used to predict the direction of drainage in an image. By following the image gradient downhill from each point in the image, the set of points which drain to each local intensity minimum can be identified. These disjoint regions are called the watersheds of the image. Similarly, the gradients can be followed uphill to local intensity maximum in the image, defining the inverse watersheds of the image.

Our first step in computing the watersheds for an image is identifying the local intensity minima. These are the points which define the bottoms of watersheds. Since an integer valued image is often a poor approximation to a smooth surface, the input image is converted to floating point and blurred using a Gaussian filter to yield a smooth image $f(x, y)$. This eliminates the plateaus in the image and simplifies the process of identifying maxima and minima. To distinguish these critical points, each pixel is compared with its eight nearest neighbors. If all neighbors are greater than the central pixel, it is identified as an intensity minimum (see Fig. 1). Similarly, all eight neighbors of an intensity maximum are less than the central pixel.

Next, we calculate the image gradient. The goal here is to identify the drainage directions for each pixel in the image. Rather than calculate the gradient based on the partial derivatives of the image, the eight neighbors of each point are searched to determine the most steeply uphill and most steeply downhill directions (i.e., the morphological gradient). These directions may or may not be in opposite directions due to discreteness. Again, the blurred floating point image 
is used to avoid problems caused by plateaus. There are nine possibilities for each of these directions (the central pixel could be an extremum), which are encoded and stored in a temporary image for use in the gradient following step of our algorithm.

Partitioning the input image into watersheds begins by marking the locations of intensity minima with unique region identifiers in an output image. For each of the remaining points in the image, the gradient information is used to follow the image downhill to some intensity minimum. The identifier of this extremum is then recorded in the output pixel corresponding to this starting point. Once all pixels in the image have been associated with their respective minima, the output image will contain the watershed regions of the image. The boundaries of watershed regions correspond to the tops of intensity ridges in the image. We locate the watershed boundaries by scanning the region image from left to right and then from top to bottom, detecting changes in watershed region numbers (see Fig. 1).

In cases where the input image contains large regions of uniform intensity, the location of intensity minima or maxima in our Gaussian smoothed images may or may not correspond to the geometric center of the original flat regions. For example, if the neighbors of a flat region decrease in intensity rapidly on the left and gradually on the right, the detected location of the local maxima will be to the right of the center of the original flat region. Fortunately, this potential for poor extrema localization has little effect on the identification of watershed regions since all points in the "plateau region" drain to a single minima regardless of its location.

One important optimization we use is to terminate this downhill search whenever we reach a pixel which has already been associated with an intensity minimum. This limits the length of our downhill search considerably. As a consequence, our watershed program is comparable in speed with other methods in the literature. For example, on a SUN SparcStation IPX, our method takes an average of $5.0 \mathrm{~s}$ to process images in our collection of 25 images. The immersion simulation approach is reported to take $6.3 \mathrm{~s}$ for $512 \times 512$ images on the same platform [42]. Faster watershed algorithms are now available [14], but we have not investigated their use in our system since gradient tracking is also used in our multiscale analysis.

Since watershed boundaries are defined in terms of the global drainage patterns of the image rather than local differential geometry, we find that not all visually apparent ridge tops in the image are marked as watershed boundaries. Only those ridges that separate drainage basins are identified. Ridges that correspond to flanks on the side of larger ridges are not detected. This may or may not be a problem, depending on the needs of the image analysis application. In very smooth images that have few intensity minima, the tops of some ridgelike structures may be missed. In more realistic images there are thousands of intensity minima and associated watershed regions. In this case, the image is oversegmented and the problem is identifying which watershed boundaries mark significant image structures. To address this problem, we consider the multiscale behavior of watersheds and their boundaries.

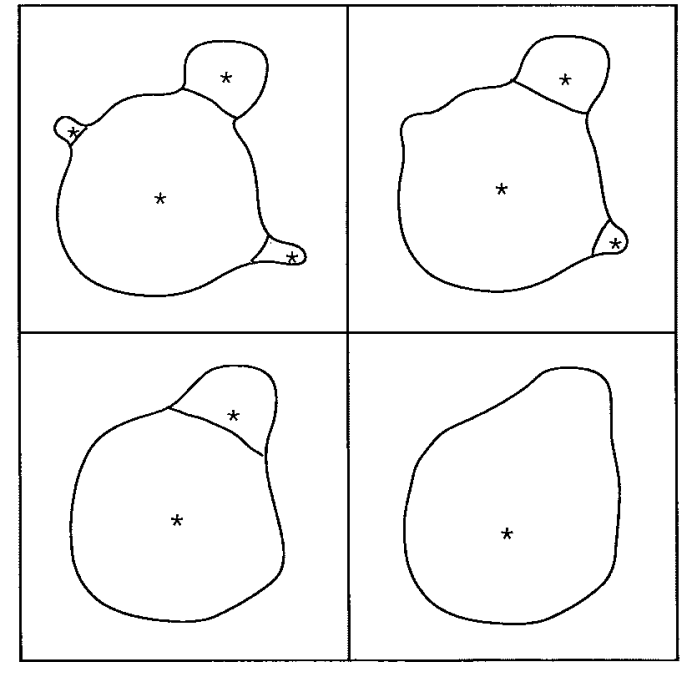

Fig. 2. Series of watershed basins and their associated intensity minima $(*)$ through scale space. As the blurring level increases from top left to bottom right, the number of minima and their associated watersheds decrease.

\section{B. Multiscale Watershed Hierarchies}

The multiscale properties of watershed boundaries depends on the multiscale behavior of the intensity extrema that define these regions. It is well known that as an image is gradually blurred with a series of Gaussians, the image structure simplifies [26], [44]. This has led to a definition of scale-space image sequence $m(x, y, \sigma)=f(x, y) * g_{\sigma}(x, y)$ where an input image $f(x, y)$ is convolved with a sequence of Gaussians with standard deviation $\sigma$. In the case of intensity extrema, we typically expect the number of maxima, minima and saddle points in $m(x, y, \sigma)$ to decrease as $\sigma$ increases. As this blurring progresses, all but one of the intensity extrema in an infinite extent image will eventually move toward a saddle point and annihilate. Watershed regions associated with these intensity extrema are annihilated at the same time (see Fig. 2).

For 2-D images, there are circumstances where intensity extrema and saddle points can also emerge as an image is Gaussian blurred [27]. Thus, watersheds can occasionally be created as scale decreases. In practice, this property of Gaussian scale-space does not cause problems in our application if the steps in scale-space $\Delta \sigma$ chosen to be moderately large (i.e., 50 steps in scale-space rather than 500 steps). As we shall see, this is a consequence of our selection of evenly spaced Gaussian blurring levels, and our method for linking regions from one scale to the next.

To impose a multiscale hierarchy on watershed regions, the paths of intensity extrema in the image must be followed as blurring proceeds. When an intensity minimum annihilates into a saddle, the water that drains toward the annihilated minimum will now drain to some other intensity minimum in the image. This defines the parent-child relationship between these two watershed regions. The region associated with the annihilated intensity minimum is said to be a subregion of the watershed region which is directly downhill from the annihilation point. By continuing this process for all intensity extrema in the image, a hierarchy on watershed regions is defined. The amount of blurring necessary to cause two regions 
to merge is related to the height and width of the ridge that initially separated the regions. This is quite different from approaches that merge based on the lowest intensity along a ridge or the average intensity.

Given the original locations of intensity extrema in the image, our extremum linking algorithm operates by following these points through a predetermined sequence of blurring levels. Rather than using the computationally expensive process of linking all image points to their isointensity counterparts from one level of blurring to the next [27], a fast heuristic is employed. Gradient descent is used to link minima from one blurring level to the next. Given an intensity minimum at position $(x, y)$ at blurring level $n$, we follow the image gradient downhill from position $(x, y)$ in level $n+1$ until another intensity minimum is encountered. This is recorded as the link from level $n$ to level $n+1$ of the former minimum.

The links defined by this process will have duplicates whenever there are fewer extrema in level $n+1$ than in level $n$. This occurs whenever local intensity extrema are annihilated. For example, if the extremum at position $(x, y)$ annihilates at blurring level $n+1$, the extremum at $(x, y)$ will be linked by gradient following to some other pixel at location $\left(x^{\prime}, y^{\prime}\right)$ in level $n+1$. At the same time, a second pixel very near $\left(x^{\prime}, y^{\prime}\right)$ in level $n$ will also be linked to $\left(x^{\prime}, y^{\prime}\right)$ in level $n+1$. To determine which link corresponds to the annihilated extremum, the lengths of all links from level to level are compared. If two or more extremum points link to the same point, the extremum with the shortest distance link is selected as the normal link, while the other links are recorded as annihilation links. Thus, each annihilated intensity extremum is linked to the extremum at the next blurring level which is directly downhill from the annihilated extremum.

Fortunately, our linking algorithm is less sensitive to the choice of blurring rate than other multiscale methods because we are only interested in building a watershed region hierarchy based on how the intensity extrema in the image are linked from level to level. Instead of using optimal blurring rates [28] to compensate for variations in the annihilation rate of critical points among images or as blurring proceeds, we use a table-driven interpolation scheme to select a sequence of $N$ Gaussian blurring standard deviations such that the percentage change in the number of minima after each blurring step remains approximately constant. Specifically, if we have $M_{\text {low }}$ intensity minima at the lowest scale $\sigma_{\text {low }}$ and $M_{\text {high }}$ at the highest scale $\sigma_{\text {high }}$, and we desire $N$ intermediate scales, then the necessary percentage change is $P=$ $\exp \left(\ln \left(M_{\text {high }} / M_{\text {low }}\right) / N\right)$. The sequence of blurring levels chosen is then $\sigma_{i}=\tilde{\sigma}\left(M_{\mathrm{low}} P^{i}\right)$ for $i \in[0 \cdots N-1]$. The lookup function $\tilde{\sigma}$ uses linear interpolation between known $(\sigma, M)$ values to return the blurring level $\sigma$ with approximately $M$ intensity minima. The choice of an appropriate value for $N$ depends on the computational power available and the desired accuracy of the watershed hierarchy. We have experimented with a variety of values and find that $N=25$ yields satisfactory results in reasonable time for most images.

It is easy to build a hierarchy on the watershed regions in an image once we have determined the annihilation level of intensity extrema and have established a parent-child rela-

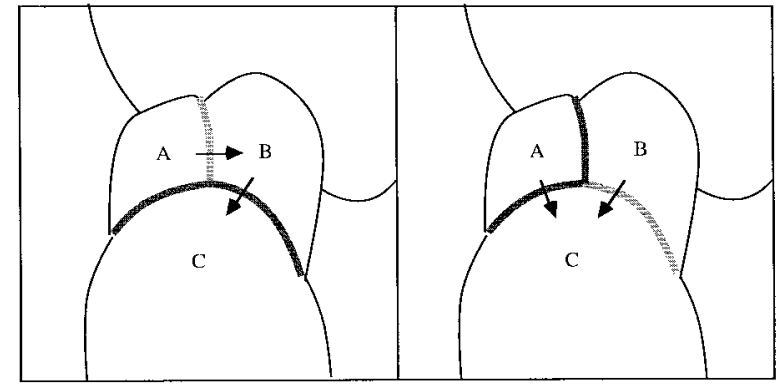

Fig. 3. Associating scale with watershed boundaries. In case (a), region A annihilates into $\mathrm{B}$ before region $\mathrm{B}$ annihilates into $\mathrm{C}$, so the $\mathrm{AB}$ boundary is assigned a low scale. In case (b), region $B$ annihilates into $C$ before region $A$ annihilates into $\mathrm{C}$, so $\mathrm{AB}$ is assigned a high scale.

tionship between their associated regions. Because we ignore the case of critical point creation, each region has a single parent region, and the relationships between regions can be described using a tree. The region associated with the final intensity minima in the image is the root of this tree. There is no limit on the number of subregions associated with any given region, so the tree describing the region hierarchy can have an arbitrary number of branches at every level. The situation is not so straightforward when we describe scale of watershed boundaries.

\section{Associating Scale with Watershed Boundaries}

Once a hierarchy has been imposed on the intensity minima that define the bottoms watershed regions, our next objective is to associate scale information with the individual curve segments that make up the watershed boundaries. With this measure of scale for each watershed boundary curve segment, it is possible to estimate the importance of each ridgelike structure in the image. Similarly, the importance of valleylike image structures can be estimated using the scale of inverse watershed boundaries.

One way to determine the scale of watershed boundaries is to interpret watershed boundaries as water barriers that disappear when adjacent watersheds annihilate into each other. Thus, if region A annihilates into region B after $N$ blurring steps, all boundary points which have both A and B as neighbors should be labeled with scale $N$. By continuing this process for all of the annihilations between adjacent regions recorded in the hierarchy table, the majority of the watershed boundary points in the image will be labeled by scale.

The remaining unlabeled boundary points correspond to situations where two adjacent regions do not annihilate directly into each other (see Fig. 3). To handle this situation, the watershed hierarchy is searched to find the lowest scale watershed region that is a parent of both of these regions. The scale of the boundary between these regions is then determined to be the highest scale required for these two regions to annihilate into the parent region. For example, if region A annihilates into region B at scale $N$ and region B annihilates into $\mathrm{C}$ at scale $M$, the scale of the boundary between $\mathrm{A}$ and $\mathrm{C}$ is equal to $M$. This corresponds to the lowest scale at which water originally in region A will mix with water from region $\mathrm{C}$. 


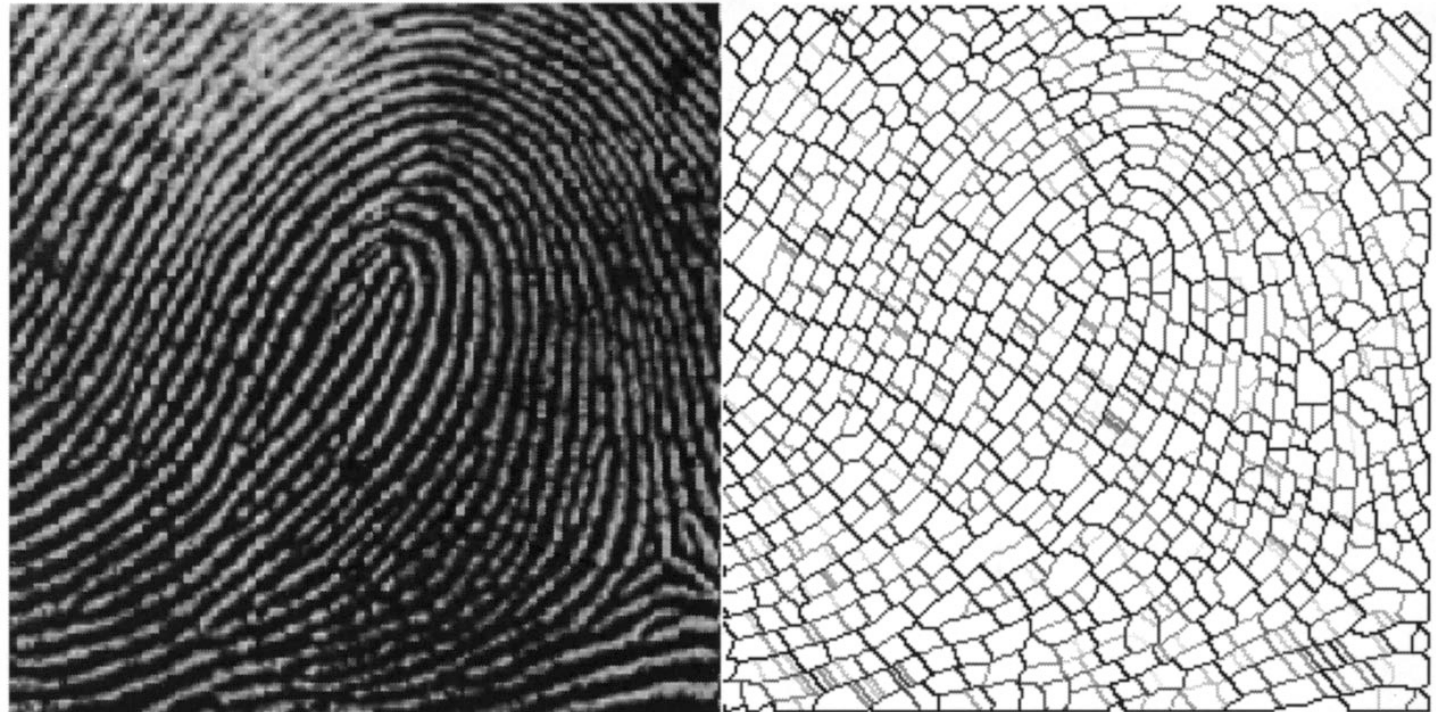

(a)

(b)

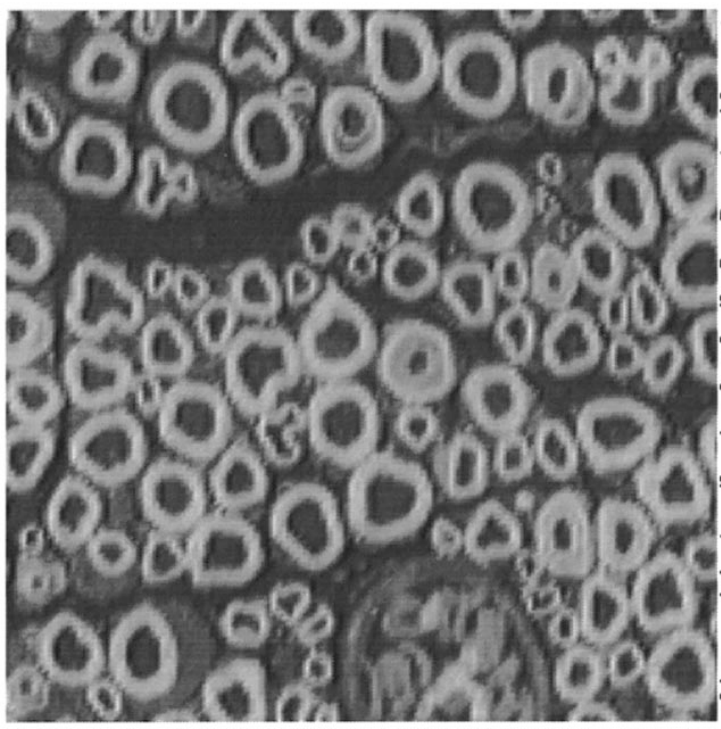

(c)

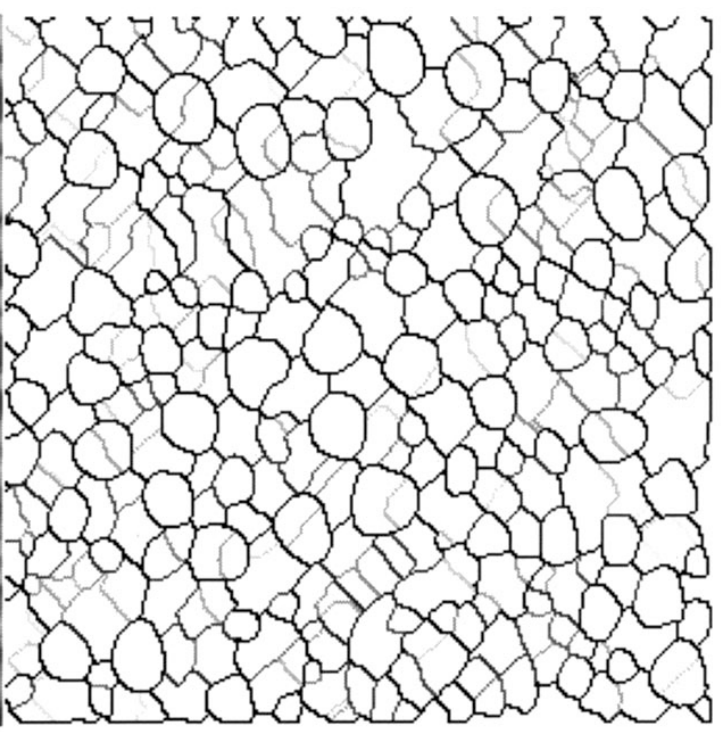

(d)

Fig. 4. (a) Finger print image. (b) Corresponding scale labeled watershed boundaries. (c) Nerve cell image. (d) Corresponding scale labeled watershed boundaries.

Our algorithm for associating scale with watershed boundary pixels has three steps: 1) scanning the watershed region from left to right and from top to bottom to detect changes in region number; 2) using the methods described above to identify the scale of the boundary between these two adjacent regions; and 3) recording this information in the output image. To reduce the computation time required to identify the scale of watershed boundaries, this information is calculated only for adjacent watershed regions, not all region pairs. Once this process is complete, the scale of all watershed boundaries are recorded in an output image with intensity proportional to scale. Displaying this grey-scale image gives an indication of the importance of individual ridges (or valleys) in the original image.

We have tested our algorithm on a number of images where the structures of interest are ridgelike or valleylike (see Fig. 4).
For images of finger prints, the size and spacing of ridges is very uniform. The scales assigned to ridge tops reflect this and are quite uniform. For images of cells in a microscopic image, watershed boundaries coincide with the outlines of cells. Again, multiscale analysis yields watershed boundaries whose scales correspond to the significance of structures in the image.

\section{GRADIENT WATERShED HIERARChIES}

Whenever objects of interest in an image are bounded by strong intensity discontinuities, it is reasonable to make use of edges based on the intensity gradient to locate objects in the image. If we consider the geometry of gradient magnitude images, we find that ridge tops mark the edges of objects in the image. Calculating the watershed regions for gradient magnitude images has therefore proven to be an effective 


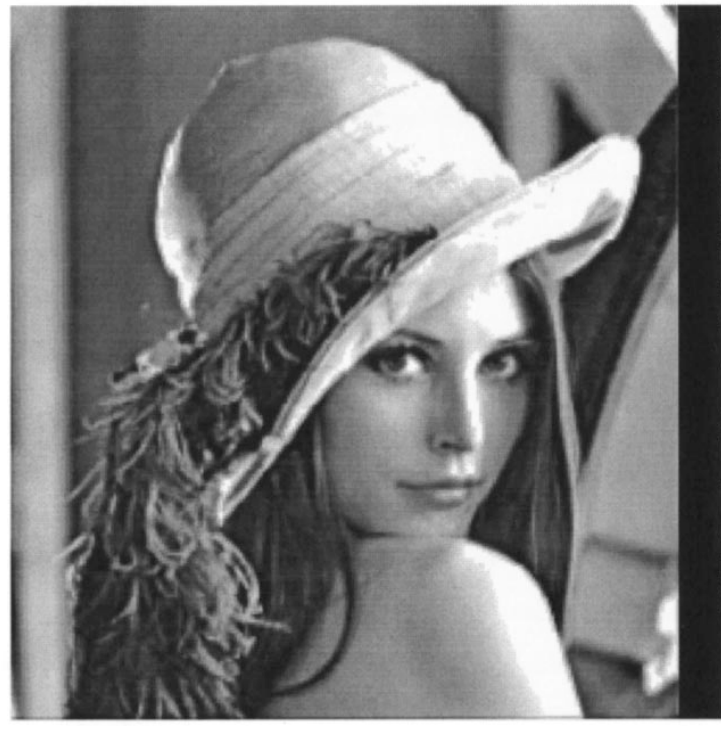

(a)

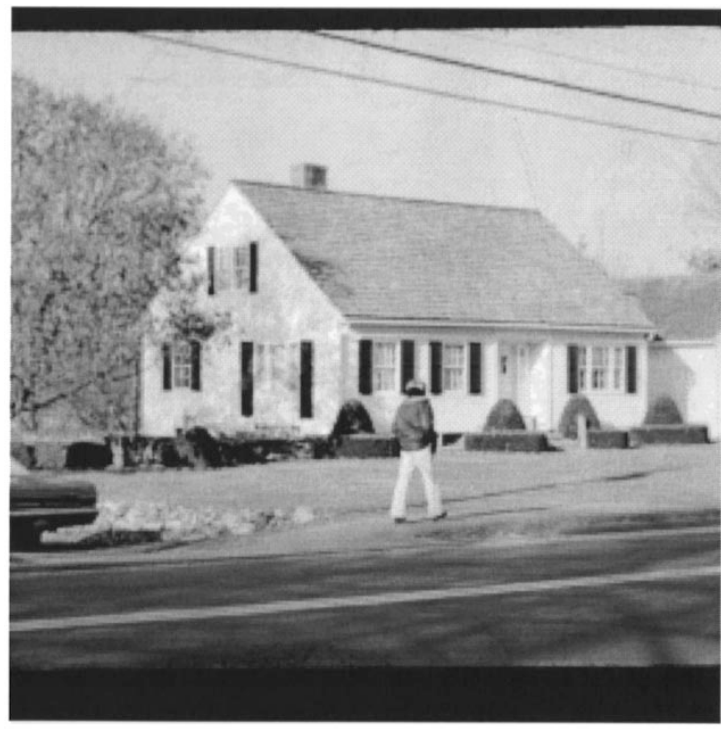

(c)

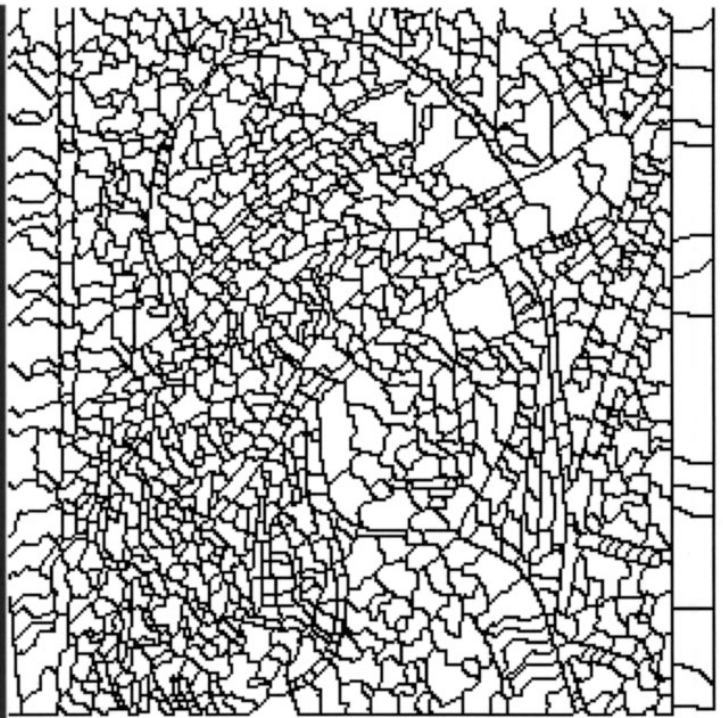

(b)

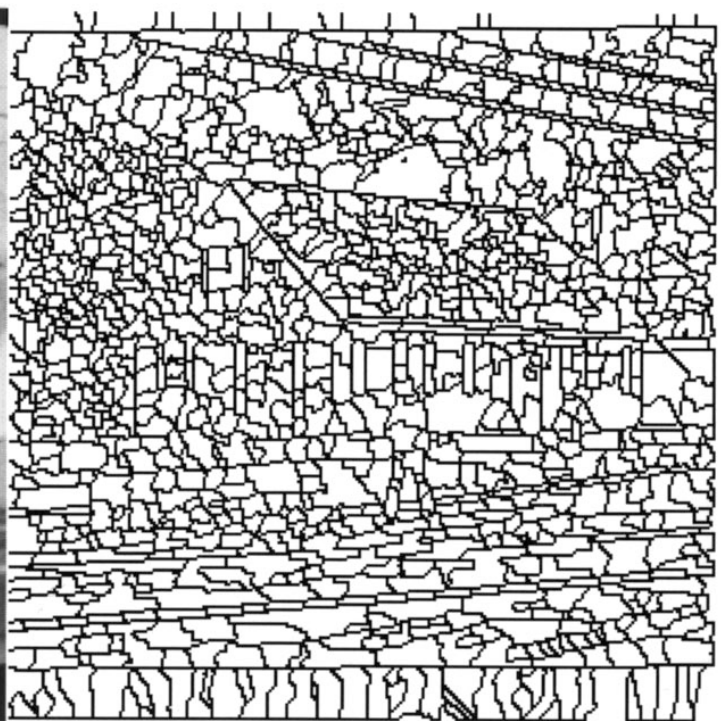

(d)

Fig. 5. (a) Face image. (b) Corresponding gradient watershed boundaries. (c) House image. (d) Corresponding gradient watershed boundaries .

means for image segmentation [3], [32]. In this section, we begin by describing how gradient watersheds are computed for a single image and then across multiple scales. This section concludes with a discussion of our interactive hierarchy based image segmentation system.

\section{A. Gradient Watersheds and Their Boundaries}

The process of computing the gradient watersheds and boundaries for a given image is quite simple. First, the gradient magnitude must be computed at every point in our floating point image $f(x, y)$. We use symmetric finite differences, but any reasonable method can be used. The resulting image is then treated as an intensity image and watersheds and their boundaries are computed using the method described in Section II. The boundaries of gradient watersheds correspond quite closely to the edges of the original image (see Fig. 5).
For face images, the boundaries of the eyes, mouth, hair, and profile can be easily seen. The boundaries of visually sensible regions are also present for typical outdoor images and common medical images.

There are some important differences between this approach and other edge-detection-based segmentation methods. First, object edges obtained by calculating gradient watershed boundaries are always guaranteed to be connected and closed. This is a consequence of the fact that watersheds are disjoint connected regions. This means that all of the boundary edges for a single object can be trivially extracted without complex tracking or connecting of edges, thereby avoiding one of the pitfalls of many edge detection methods.

Second, edges that correspond to cracks in objects or texture within objects will not be output as gradient watershed boundaries. This is also a consequence of the fact that watersheds are disjoint connected regions. This point may or may not be 
a problem for subsequent image analysis tasks. Quantitative analysis such as computing areas or volumes will not be affected. On the other hand, qualitative tasks such as detecting fractures in structures may not be possible without additional edge detection.

Third, gradient watershed regions can be used to interactively construct the image region associated with an object of interest. Since these regions do not to cross significant edges in the original image, smaller building units are not necessary. In very smooth images, this object segmentation scheme will often be satisfactory, but in more realistic images there will be thousands of gradient watershed regions due to oversegmentation. Objects of interest in the image may consist of dozens of regions. To construct larger object regions, we have developed a scale-based hierarchy on these regions.

\section{B. Multiscale Gradient Watershed Hierarchies}

To build a scale-based hierarchy on gradient watershed regions, we need to consider the multiscale properties of the image gradient. The major question is how and when should the image be smoothed. A natural choice is to apply Gaussian blurring before computing the gradient magnitude. Here, the input image $f(x, y)$ is convolved with a Gaussian $g_{\sigma}(x, y)$ with standard deviation $\sigma$ to obtain a multiscale image sequence. The gradient magnitude at each scale is then calculated to yield a multiscale image sequence $m(x, y, \sigma)=$ $\left\|\nabla f(x, y) * g_{\sigma}(x, y)\right\|$. It is well known that the positions of edges in an image change position as scale changes [2], [30]. As blurring increases, edges associated with small objects disappear and the corners of large objects are rounded. Edges associated with long narrow objects in the image often spread apart as the object is blurred. Ridges in the $m(x, y, \sigma)$ image change position and height to reflect this behavior.

The multiscale behavior of intensity minima in the gradient magnitude image is less obvious. Intensity minima in the gradient magnitude image occur near the centers of homogeneous regions in the original image. As blurring increases, the edges separating homogeneous regions are gradually removed, and one of the intensity minima associated with these regions is annihilated. Intensity minima surrounded by taller and wider image edges persist longer in scale-space, so the amount of blurring necessary to cause extrema annihilation has a natural correspondence with feature size.

Although it is possible to derive a relationship between blurring level and the number of intensity extrema in an image [28] the exact relationship between blurring level and the number of extrema in the gradient magnitude image is very difficult to predict. Rather than doing so, we have pragmatically selected a sequence of $N$ blurring levels which cause a fixed percentage $P$ of the intensity minima in the gradient magnitude image to be annihilated at each blurring step. Specifically, if we have $M_{\text {low }}$ gradient minima at the lowest scale $\sigma_{\text {low }}$ and $M_{\text {high }}$ at the highest scale $\sigma_{\text {high }}$, and we desire $N$ intermediate scales, then the necessary percentage change is $P=\exp \left(\ln \left(M_{\text {high }} / M_{\text {low }}\right) / N\right)$. The sequence of blurring levels chosen is then $\sigma_{i}=\tilde{\sigma}\left(M_{\text {low }} P^{i}\right)$ for $i \in$ $[0 \cdots N-1]$. The lookup function $\tilde{\sigma}$ uses linear interpolation

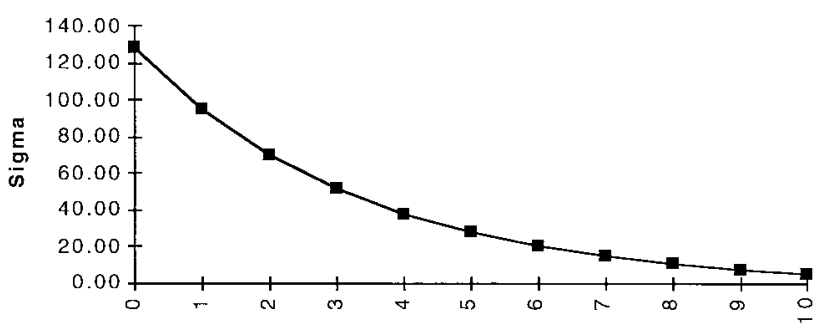

(a)

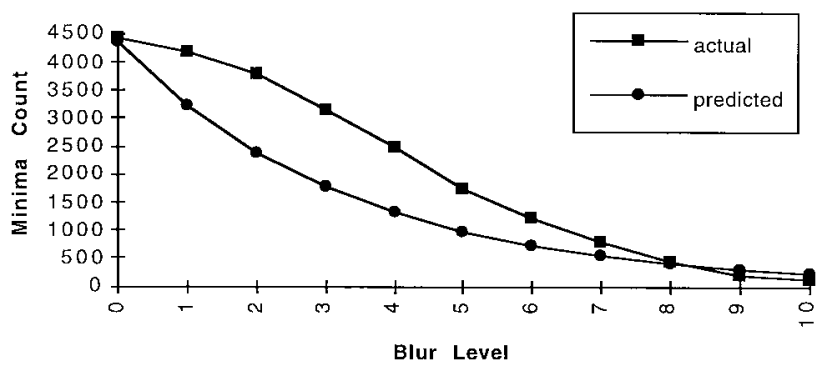

(b)

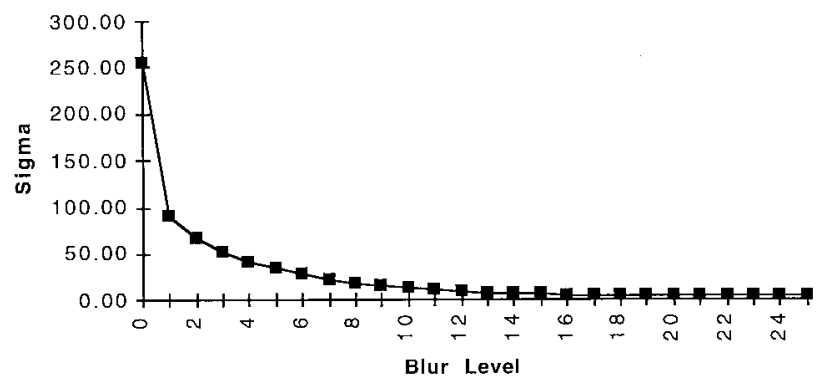

(c)

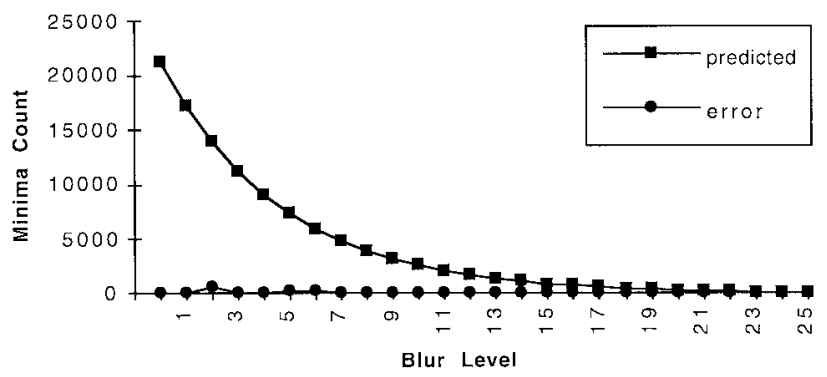

(d)

Fig. 6. (a) Ten automatically selected of blurring levels. (b) Corresponding number of gradient magnitude minima at each scale (bottom) for a typical $256 \times 256$ image. The interpolation table used to model the $\sigma(\mathrm{m})$ function consisted of only two values, so there is considerable difference between the predicted and actual number of minima at each scale. (c) Twenty-five computed blurring levels (d) Corresponding number of gradient magnitude minima at each scale (bottom) for a typical $512 \times 512$ image. An interpolation table with five entries produced an average difference between the actual and predicted number of minima of less than $6 \%$.

between known $(\sigma, M)$ values to return the blurring level $\sigma$ with approximately $M$ minima in the gradient magnitude image. When only two $(\sigma, M)$ values are used to define $\tilde{\sigma}$, we can expect considerable error. When between five and ten $(\sigma, M)$ values are used, there is almost no difference between the number of predicted gradient magnitude minima and the number of minima detected (see Fig. 6). 


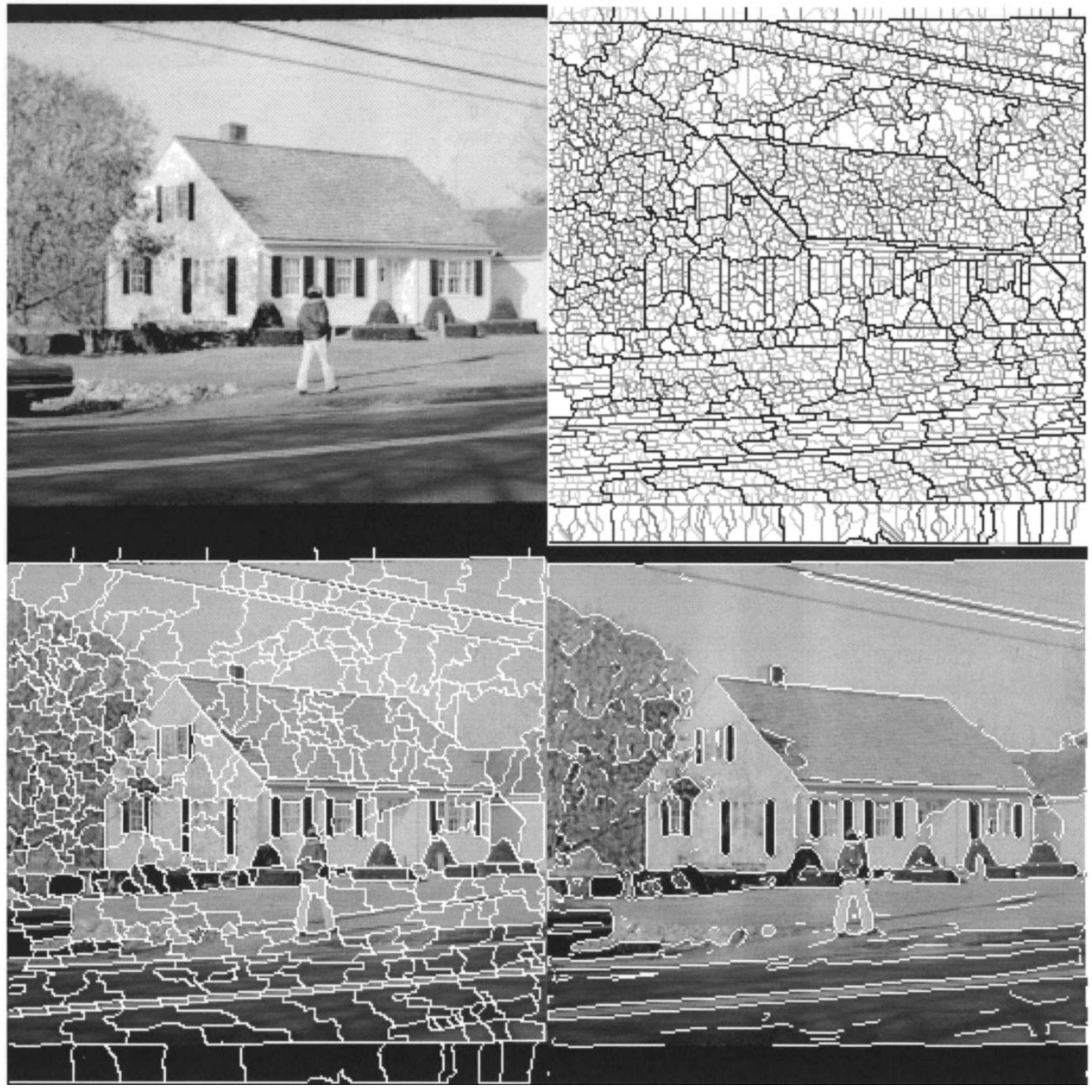

(a)

Fig. 7. (a) House image (top left), scale labeled gradient watershed boundaries (top right), high scale (sigma $=10)$ gradient watershed boundaries $($ bottom left), high scale (sigma $=20$ ) Canny edges (bottom right).

Fine sampling of scale-space reduces the chance of multiple watershed regions merging into a single parent region when constructing the region hierarchy. Coarse sampling requires less computation time and often yields acceptable region hierarchies. In practice, the choice of number of blurring levels $N$ depends on the type of image being processed and desired segmentation accuracy. For example, if we know that each watershed region has approximately $K$ adjacent regions, selecting $N$ and $P$ such that $P=1 / K$ would result in each parent merging with a single child most of the time. We have experimented with $N$ values between 5 and 100, and found that $N=25$ yields satisfactory segmentation results for images in our collection.

The intensity minima of $m(x, y, \sigma)$ are linked from one level of blurring to the next and used to construct a scale-based hierarchy on the gradient watershed regions. This hierarchy was then used to compute the scales associated the corresponding region boundaries using the technique described in Section II. The result is very promising. For a variety of natural and med- ical images, the scale labeled watershed boundaries typically correspond with visually sensible image edges (see Fig. 7). Edges of large object features have higher scales (shown by brighter lines) than edges of small scale image features (shown by darker lines). We have found that by simply thresholding this boundary scale image, it is often possible to obtain the outlines of objects of interest in an image.

\section{Interactive Image Segmentation}

Once an image has been decomposed into visually sensible atomic regions and a meaningful hierarchy has been imposed on these regions, the process of segmenting an image is greatly simplified. Users can point to objects of interest and use the hierarchy to help them combine atomic regions to construct the image region associated with these objects. Our current system has one window where the original image is displayed and an adjacent window where the object being segmented is displayed. In a third window, we display the scale-labeled 


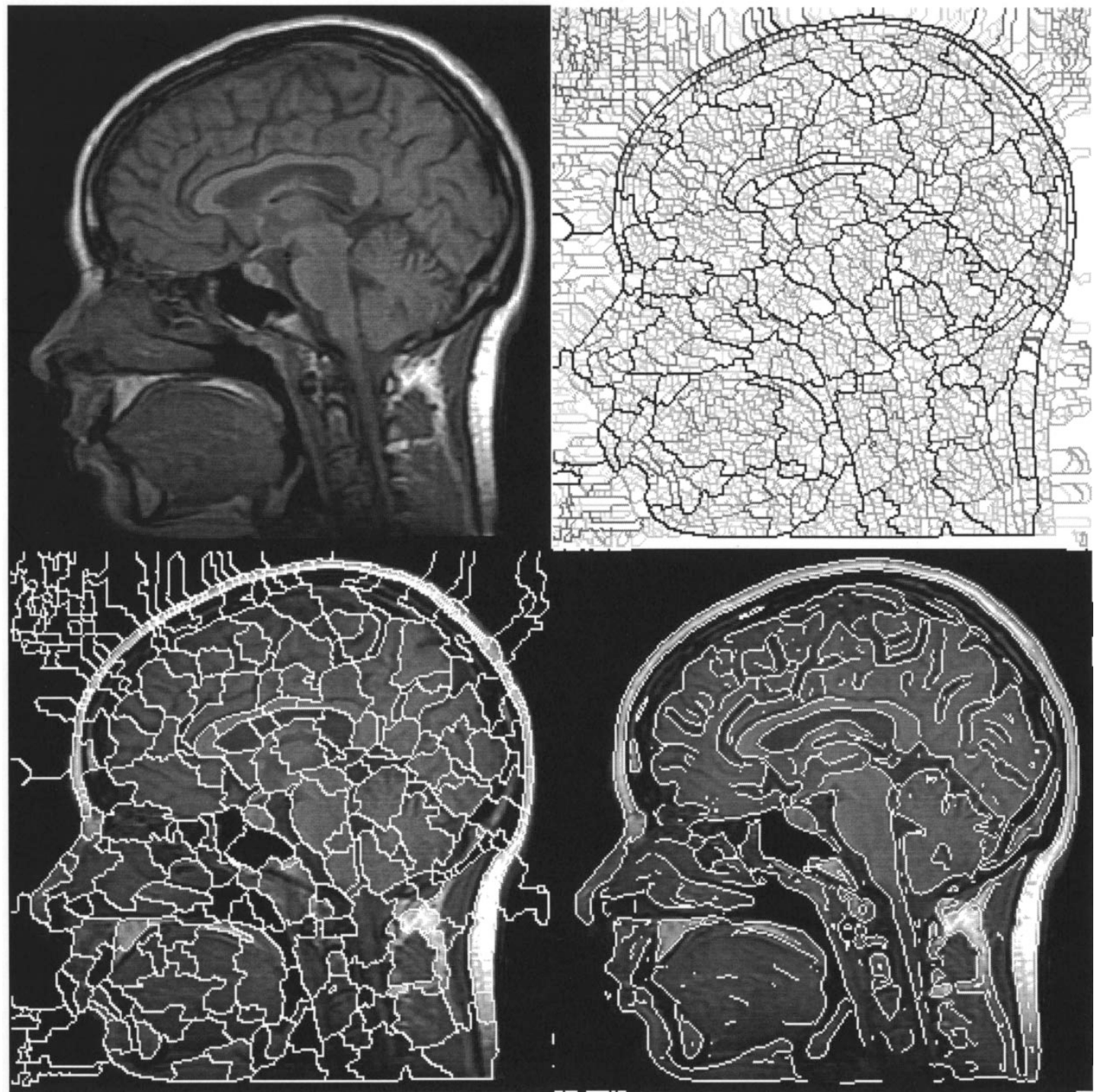

(b)

Fig. 7. (Continued.) (b) MRI brain image (top left), scale labeled gradient watershed boundaries (top right), high scale (sigma $=10)$ gradient watershed boundaries (bottom left), high scale (sigma $=20$ ) Canny edges (bottom right).

watershed boundaries computed from our region hierarchy. Our system supports two interactive segmentation modes: region painting and hierarchy traversal.

In region painting mode, the mouse is used to manually select points within an object of interest. The inverse mapping from $(x, y)$ coordinates is used to identify the selected region number. All image points within this region can then be displayed. By holding the mouse button down and moving to adjacent regions, additional atomic regions can be simultaneously displayed. Unwanted regions can be deleted from the object in a similar manner. Thus, the user can "paint" the object of interest using watershed regions as building blocks. Once the initial segmentation has been completed, additional cleaning up of the boundary could be performed using a pixel level paint brush. This system is always guaranteed to work, but can become tedious. To reduce the number of region selections necessary, we make use of watershed hierarchy.

In hierarchy traversal mode, the mouse is again used to select watershed regions. The user can then move up the region hierarchy to "grow" larger regions. Two choices are possible: 1) the user can move up one blurring level at a time and display all regions which merge into the current region at blurring levels less than or equal to the current level; or 2) the user can move up to the parent region of the current region by following the "annihilation link" and display all regions that merge into the parent region below the corresponding annihilation level. It is also possible to move down the hierarchy by reversing the operations above. For a variety of images in our collection, this approach enables users to specify objects of interest with very little user interaction (see Fig. 8).

\section{CONCLUSION}

In this paper, we have focused on the multiscale properties of watershed boundaries and gradient watershed boundaries for an image. We have described how scale-based watershed region hierarchies can be created by following isolated extrema through scale-space and how these hierarchies can be used 

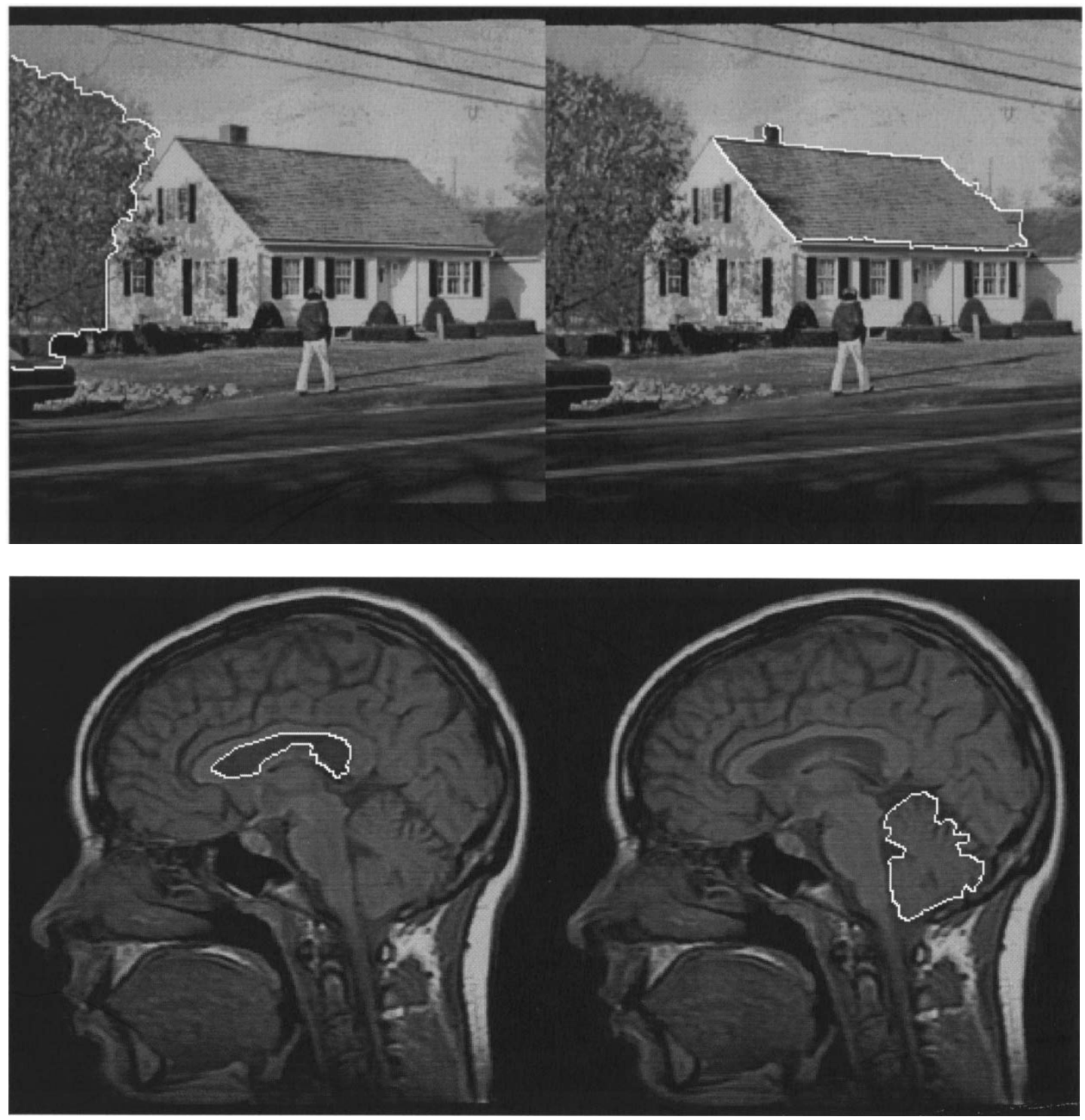

Fig. 8. Interactive segmentation using region painting and hierarchy traversal for a house image (top) and an MRI brain image (bottom). The regions shown were all specified using between two and ten mouse clicks.

for multiscale edge analysis and interactive image segmentation. The three main advantages of this approach are as follows.

1) Multiscale analysis of watershed regions is fast and easy. There is a one-to-one relationship between intensity extrema and watershed regions in the image. By exploiting this relationship, we can build hierarchies on watershed regions by simply following intensity extrema through scale-space and detecting their annihilations. This is much easier than following curve segments associated with edges or ridge tops through multiple scales and imposing a scale-based hierarchy on these structures.

2) We can associate visually sensible measurements of importance to individual curve segments which make up the boundaries of watershed regions. For images with ridgelike structures, these scale labeled watershed boundaries mark the tops of ridges. For gradient magnitude images, these curve segments correspond to edges of objects in the original image. Thus, multiscale watershed analysis can be used indirectly as an edge detection method.

3) Interactive image segmentation tools can be constructed which use gradient watershed region hierarchies to quickly and easily identify image regions associated with objects of interest. The use of region painting and hierarchy traversal are general methods that could be used with any region hierarchy of visually sensible regions.

While the advantages of this multiscale image segmentation and analysis approach are evident by our results, there are several interesting questions that remain to be investigated. Of primary interest is the mechanism for imposing scale-space. We are presently evaluating the use of anisotropic diffusion [36], [43] as an alternative to Gaussian blurring because of its edge-preserving properties. In particular, we are investigating how this smoothing method effects the motion and annihilation 
of intensity minima in the gradient magnitude image, and how this effects the resulting watershed hierarchy.

\section{REFERENCES}

[1] J. Babaud, A. P. Witkin, M. Baudin, and R. O. Duda, "Uniqueness of the Gaussian kernel for scale-space filtering," IEEE Trans. Pattern Anal. Machine Intell., vol. PAMI-8, pp. 26-33, Jan. 1986.

[2] F. Bergholm, "Edge focusing," IEEE Trans. Pattern Anal. Machine Intell., vol. 9, pp. 726-741, Nov. 1987.

[3] S. Buecher, "Watersheds of functions and picture segmentation," in Proc. IEEE Int. Conf. Acoustics, Speech, and Signal Processing, Paris, France, May 1982, pp. 1928-1931.

[4] S. Beucher and F. Meyer, "The morphological approach to segmentation: The watershed transformation," Mathematical Morphology in Image Processing, E. R. Dougherty, Ed. New York: Marcel Dekker, 1993, pp. 433-481.

[5] A. Bleau, J. DeGuise, and A. R. LeBlanc, "A new set of fast algorithms for mathematical morphology," CVGIP: Image Understand., vol. 56, pp. 178-209, Sept. 1992.

[6] J. Blom, "Topological and geometrical aspects of image structure," Ph.D. dissertation, Utrecht Univ., Utrecht, The Netherlands, 1992.

[7] P. J. Burt, T. H. Hong, and A. Rosenfeld, "Segmentation and estimation of image region properties through cooperative hierarchical computation," IEEE Trans. Syst., Man, Cybern., vol. SMC-11, pp. 802-809, Dec. 1981.

[8] J. F. Canny, "A computational approach to edge detection," IEEE Trans. Pattern Anal. Machine Intell., vol. PAMI-8, pp. 679-698, 1986.

[9] A. Cayley, "On contour and slope lines," London, Edinburgh, Dublin Philosoph. Mag. J. Sci., vol. 18, pp. 264-268, Oct. 1859.

[10] M. H. Chen and P. F. Yan, "A multiscaling approach based on morphological filtering," IEEE Trans. Pattern Anal. Machine Intell., vol. 11, pp. 649-700, 1989

[11] J. J. Clark, "Singularities of contrast functions in scale space," in Proc. 1st Int. Conf. Computer Vision, London, U.K., 1987, pp. 491-495.

[12] S. H. Collins, "Terrain parameters directly from a digital terrain model," Canad. Surv., vol. 29, pp. 507-518, 1975.

[13] H. Digabel and C. Lantuejoul, "Iterative algorithms," in Proc. 2nd Europ. Symp. Quantitative Analysis of Microstructures in Material Science, Biology, and Medicine, Caen, France, Oct. 1978, pp. 85-99.

[14] B. P. Dobrin, T. Viero, and M. Gabbouj, "Fast watershed algorithms: Analysis and extensions," Nonlinear Image Processing V. San Jose, CA: SPIE, pp. 209-220, 1994.

[15] J. M. Gauch and S. M. Pizer, "Image description via the multiresolution intensity axis of symmetry," in Proc. 2nd Int. Conf. Computer Vision, Tampa, FL, Dec. 1988, pp. 269-274.

[16] - "Multiresolution analysis of ridges and valleys in grey-scale images," IEEE Pattern Anal. Machine Intell., vol. 15, pp. 635-646, June 1993.

[17] T. Geraud, J. F. Mangin, I. Bloch, and H. Maitre, "Segmenting the internal structures in 3D MR images of the brain by Markovian relaxation on a watershed based adjacency graph," in Proc. IEEE Int. Conf. Image Processing, Washington, DC, 1995, vol. 3, pp. 548-551.

[18] L. D. Griffin, A. F. C. Colchester, and G. P. Robinson, "Scale and segmentation of grey-level images using maximum gradient paths," in Proc. Information Processing in Medical Image Conf., Wye, U.K., 1991, pp. 256-272.

[19] M. W. Hansen and W. E. Higgins, "Watershed-driven relaxation labeling for image segmentation," IEEE Int. Conf. Image Processing, Austin, TX, 1994, pp. 460-464

[20] R. M. Haralick, "Zero-crossing of second directional derivative edge operator," IEEE Trans. Pattern Anal. Machine Intell., vol. PAMI-6, pp. 58-68, Jan. 1984

[21] R. M. Haralick, S. Sternberg, and X. Zhuang, "Image analysis using mathematical morphology," IEEE Pattern Anal. Machine Intell., vol. PAMI-9, pp. 532-550, July 1987.

[22] R. A. Hummel, "Representations based on zero crossings in scalespace," in Proc. IEEE Conf. Computer Vision and Pattern Recognition, Miami Beach, FL, June 1986, pp. 204-209.

[23] P. Jackway, "Morphological scale space," in Proc. 11th IAPR Int. Conf. Pattern Recognition, The Hague, The Netherlands, 1992, pp. 252-255.

[24] _ "Morphological multiscale gradient watershed image analysis," 9th Scand. Conf. Image Analysis, June 6-9, 1995.
[25] __ "Gradient watersheds in morphological scale-space," IEEE Trans. Image Processing, vol. 5, pp. 913-921, June 1996.

[26] J. J. Koenderink, "The structure of images," Biol. Cybern., vol. 50, pp. $363-370,1984$

[27] L. M. Lifshitz, "Image segmentation using global knowledge and a priori information," Ph.D. dissertation, Univ. North Carolina, Chapel Hill, 1987.

[28] T. Lindeberg, "Discrete scale-space theory and the scale space primal sketch," Ph.D. dissertation, Royal Inst. Technol., Stockholm, Sweden, May 1991.

[29] D. Marks, J. Dozier, and J. Frew, "Automated basin delineation from digital elevation data," Geoprocessing, vol. 2, pp. 299-311, 1984.

[30] D. Marr and E. Hildrith, "Theory of edge detection," in Proc. R. Soc. Lond. B, vol. 207, pp. 187-217, 1980.

[31] J. C. Maxwell, "On hills and dales," The London, Edinburgh, Dublin Philosoph. Mag. J. Sci., 4th Series, vol. 40, pp. 421-425, Dec. 1870.

[32] F. Meyer and S. Beucher, "Morphological segmentation," J. Vis. Commun. Image Represent., vol. 1, pp. 21-46, Sept. 1990.

[33] F. Meyer, "Topographic distance and watershed lines," in Proc. 1st Int. Workshop on Mathematical Morphology and its Applications to Signal Processing, Barcelona, Spain, July 1994, pp. 113-125.

[34] B. Ogor, V. Haese-Coat, and K. Kpalma, "Cooperation of mathematical morphology and region growing for remote sensing region growing," Image Signal Process. Remote Sensing II. vol. 2579, pp. 375-386, 1995.

[35] D. W. Paulus, Greiner, and Torsten, "Watershed transformation on time series of medical thermal images," SPIE: Intell. Robots Comput. Vis. XIV, Philadelphia, PA, pp. 700-711, 1995.

[36] P. Perona and J. Malik, "Scale space and edge detection using anisotropic diffusion," IEEE Trans. Pattern Anal. Machine Intell., vol. 12, pp. 629-639, July 1990.

[37] T. K. Puecker and D. H. Douglas, "Detection of surface specific points by local parallel processing of discrete terrain elevation data," Comput., Vis., Graph., Image Process., vol. 4, pp. 375-387, 1975.

[38] A. Rosenfeld and M. Thurston, "Edge and curve detection for visual scene analysis," IEEE Trans. Comput., vol. C-20, pp. 562-569, 1971.

[39] K. Saarinen, "Color image segmentation by a watershed algorithm and region adjacency graph processing," in Proc. IEEE Int. Conf. Image Processing, Austin, TX, 1994, pp. 1021-1025.

[40] P. Salembier and J. C. Serra, "Morphological multiscale image segmentation," in Proc. Visual Communications and Image Processing '92, Boston, MA, pp. 620-631.

[41] J. C. Serra, Image Analysis and Mathematical Morphology. New York: Academic, 1982

[42] L. Vincent and P. Soille, "Watersheds in digital spaces: An efficient algorithm based on immersion simulations," IEEE Trans. Pattern Anal. Machine Intell., vol. 13, pp. 583-598, 1991.

[43] R. T. Whitaker and S. M. Pizer, "A multi-scale approach to nonuniform diffusion," CVGIP: Image Understand., vol. 57, pp. 99-110, Jan. 1993.

[44] A. Witkin, "Scale-space filtering," in Proc. Int. Joint Conf. Artificial Intelligence, Karlsruhe, Germany, 1983, pp. 1019-1022.

[45] A. L. Yuille and T. Poggio, "Scaling theorems for zero-crossings," Memo 722, Mass. Inst. Technol., Cambridge, June 1983.

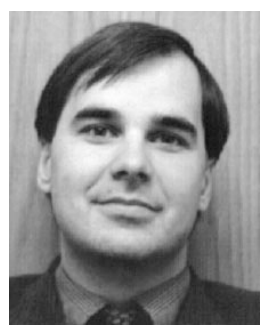

John M. Gauch (S'84-M'89) received the B.Sc. (honors) and M.Sc. (honors) degrees from Queen's University, Ont., Canada, in 1981 and 1982, respectively, and the $\mathrm{Ph} . \mathrm{D}$. degree from the University of North Carolina, Chapel Hill, in 1989, all in computer science.

From 1989 to 1993, he was an Assistant Professor in the College of Computer Science at Northeastern University, Boston, MA. In 1993, he joined the Department of Electrical Engineering and Computer Science at the University of Kansas, Lawrence, where he is now an Associate Professor. His research interests are in computer vision and digital image processing, focusing on image segmentation and motion analysis. Specific applications include tracking cells in microscopic images, classifying ice types in remote sensing, temporal segmentation of video sequences, and segmentation of multispectral images (color and medical). 